cal $M$. tuberculosis genotypic patterns, and performed tuberculin skin testing (TST) and case finding for possible exposed contacts.

Only patients 1 and 2 had identical genotypic patterns. Neither patient had baseline TST results available. Patient 1 had clinical evidence of infectiousness 3 months before the diagnosis of TB was ascertained. Among employee contacts of patient 1 , TST conversions occurred in $1(2 \%)$ of 59 , $2(6 \%)$ of $34,2(6 \%)$ of 32 , and $0(0 \%)$ of 8 who were tested at hospitals $\mathrm{A}, \mathrm{B}$, and $\mathrm{C}$ and at the residential facility, respectively. Among the other patients who were exposed to patient $1,1(3 \%)$ of $31,1(3 \%)$ of $30,0(0 \%)$ of 40 , and $12(9 \%)$ of 136 who were tested had positive TSTs at hospitals A, B, and $C$ and at the residential facility, respectively. The authors concluded that delayed $\mathrm{TB}$ diagnosis in two patients with leukemia resulted in the transmission of $M$. tuberculosis to 19 patients and staff at three hospitals and a residential facility. Baseline TB screening and earlier clinical recognition of active disease could reduce healthcare facility-associated transmission of $M$. tuberculosis among patients with malignancy.

FROM: Malone JL, $\operatorname{ljaz} \mathrm{K}$, Lambert $\mathrm{L}$, et al. Investigation of healthcare-associated transmission of Mycobacterium tuberculosis among patients with malignancies at three hospitals and at a residential facility. Cancer 2004;101:2713-2721.

\section{Nurses' Working Conditions Have Implications for Infectious Disease}

Stone et al. recently published a review of the research related to staffing patterns. They found that nurses' working conditions are risk factors for healthcare-associated infections as well as occupational injuries.

The research revealed that staffing shortages, especially those involving nurses, have been identified as one of the major factors expected to constrain the ability of hospitals to deal with future outbreaks of emerging infections.

The authors noted that nursing is a predominately female occupation for which the working conditions are often poor. Such conditions contribute to recruitment and retention problems. These factors, together with demographic changes, have resulted in a shortage of qualified nurses. Mounting evidence demonstrates that the lack of an adequate supply of qualified nurses is a global public safety issue that may require a multi-pronged policy approach. Monitoring and improving the working conditions of nurses is likely to improve the quality of healthcare by decreasing the incidence of many infectious diseases, assisting in retaining qualified nurses, and encouraging men and women to enter the profession. The authors conclude that further research is needed to understand how best to protect patients and healthcare workers. Changes in the work force will have implications for infectious disease, infection control, and occupational health professionals with a need for much more thorough training of nonprofessionals in critical practices.

FROM: Stone PW, Clarke SP, Cimiotti J, Correa-deAraujo R. Nurses' working conditions: implications for infectious disease. Emerg Infect Dis 2004;10:1984-1989.

\section{Emergence of New Strains of Methicillin-Resistant Staphylococcus aureus in a Neonatal Intensive Care Unit}

Genetically distinct strains of methicillin-resistant Staphylococcus aureus (MRSA) of community rather than hospital origin have emerged in many areas of the United States. Healy et al., from Baylor College of Medicine, Houston, Texas, conducted a study to determine whether MRSA strains causing bacteremia in infants treated from birth in a neonatal intensive care unit (NICU) demonstrated the genetic traits of community-associated MRSA. They conducted a retrospective cohort study among NICU infants with bacteremia due to MRSA during 2003 in a NICU of a large tertiary-care center in Houston. MRSA isolates were characterized by antimicrobial susceptibility testing and staphylococcal cassette chromosome mec (SCCmec) typing by polymerase chain reaction. All MRSA cases were reviewed for clinical severity of infection and outcome.

During 2003, a total of 8 (47\%) of 17 infants with bacteremia due to $S$. aureus had MRSA infection. Isolates from $6(75 \%)$ of these 8 infants carried the SCCmec genes (class $\mathrm{B} m e c$ and $c(r 2)$ that are characteristic of community MRSA; 4 isolates were type IVa. All 6 isolates were resistant to beta-lactam antibiotics and erythromycin; 1 was also resistant to clindamycin. One isolate could not be typed, and another carried the SCCmec type II gene (typical of hospital-associated strains) and was susceptible only to vancomycin. Seven (88\%) of 8 infants presented with septic shock. Despite initial treatment with vancomycin, $3(38 \%)$ of the infants died, and 3 of the infants who survived had complications requiring prolonged antimicrobial therapy; these 6 infants had MRSA isolates with genetic characteristics of isolates of community origin. The authors concluded that community-associated MRSA strains have emerged as a significant cause of nosocomial sepsis in neonates hospitalized in the NICU since birth and have caused disseminated infection with substantial morbidity and mortality.

FROM: Healy CM, Hulten KG, Palazzi DL, Campbell JR, Baker CJ. Emergence of new strains of methicillin-resistant Staphylococcus aureus in a neonatal intensive care unit. Clin Infect Dis 2004;39:1460-1466. 